\title{
Streptomyces harenosi sp. nov., a home for a gifted strain isolated from Indonesian sand dune soil
}

\author{
Ali Budhi Kusuma ${ }^{1,2}$, Imen Nouioui ${ }^{1,3}$, Hans-Peter Klenk ${ }^{1}$ and Michael Goodfellow ${ }^{1, *}$
}

\begin{abstract}
A polyphasic study was undertaken to establish the position of a Streptomyces strain, isolate PRKS01-65 ${ }^{\top}$, recovered from sand dune soil collected at Parangkusumo, Yogyakarta Province, Java, Indonesia. A combination of chemotaxonomic, cultural and morphological properties confirmed its position in the genus of Streptomyces. Comparative $16 \mathrm{~S}$ rRNA gene sequence analyses showed that the isolate was most closely related to Streptomyces leeuwenhoekii C34 ${ }^{\top}$ (99.9\% similarity) and loosely associated with the type strains of Streptomyces chiangmaiensis (98.7\% similarity) and Streptomyces glomeratus (98.9\% similarity). Multilocus sequence analyses based on five conserved housekeeping gene alleles confirmed the close relationship between the isolate and S. leeuwenhoekii $\mathrm{C} 34^{\top}$, although both strains belonged to a well-supported clade that encompassed the type strains of S. glomeratus, Streptomyces griseomycini, Streptomyces griseostramineus, Streptomyces labedae, Streptomyces lomondensis and Streptomyces spinoverrucosus. A comparison of the draft genome sequence generated for the isolate with corresponding whole genome sequences of its closest phylogenomic neighbours showed that it formed a well-separated lineage with S. leeuwenhoekii $\mathrm{C} 34^{\top}$. These strains can also be distinguished using a combination of phenotypic properties and by average nucleotide identity and digital DNA-DNA hybridization similarities of 94.3 and $56 \%$, values consistent with their classification in different species. Based on this wealth of data it is proposed that isolate PRKS01-65 $\left(=N C I M B 15211^{\top}=C C M M B 1302^{\top}=\right.$ ICEBB${ }^{\left.03^{\top}\right)}$ be classified as Streptomyces harenosi sp. nov. The genome of the isolate contains several biosynthetic gene clusters with the potential to produce new natural products.
\end{abstract}

\section{INTRODUCTION}

The emended family Streptomycetaceae $[1,2]$ includes the genus Streptomyces [1], the type genus [1] and five other genera. The genus Streptomyces encompasses over 800 species with validly published names (www.bacterio.net. streptomyces.html) most of which have been assigned to multi- and single-membered phyletic lines in Streptomyces $16 \mathrm{~S}$ rRNA gene trees $[3,4]$. Genera belonging to the family
Streptomycetaceae can be distinguished using a combination of genomic, genotypic and phenotypic features $[3,4]$.

Streptomycetes are best known as a source of new antibiotics, anti-cancer and other specialized (secondary) metabolites $[5,6]$, hence the continued interest in them as a source of novel drugs needed in the fight against multi-drug resistant microbial pathogens [7, 8]. Previously unknown Streptomyces strains isolated from deep-sea sediments and desert soils, using taxonomic approaches to drug discovery $[9,10]$, are a

Author affiliations: 'School of Natural and Environmental Sciences, Ridley Building 2, Newcastle University, Newcastle upon Tyne NE1 7RU, UK; ${ }^{2}$ Indonesian Centre for Extremophile Bioresources and Biotechnology (ICEBB), Faculty of Biotechnology, Sumbawa University of Technology, Sumbawa Besar, 84371, Indonesia; ' 2 eibniz-Institut DSMZ - German Collection of Microorganisms and Cell Cultures, Inhoffenstraße 7B, 38124 Braunschweig, Germany.

*Correspondence: Michael Goodfellow, michael.goodfelloow@ncl.ac.uk

Keywords: Streptomyces harenosisp. nov; arid sandy soil; polyphasic taxonomy; genomics.

Abbreviations: AntiSMASH, antibiotics and secondary metabolite analysis shell; atpD, ATP synthase F1-subunit; BGCs, biosynthetic gene clusters; dDDH, digital DNA-DNA hybridization; GBDP, genome BLAST distance phylogeny; GGDC, genome-to-genome distance calculator; gyrB, gyrase B subunit; ISP, International Streptomyces Project; MLSA, multilocus sequence analyses; orthoANI, orthologous average nucleotide identity; RAST, rapid annotation using subsystem technology; recA, recombinase protein A; rpoB, DNA-directed RNA polymerase B subunit; trpB, tryptophan synthase B subunit; TYGS, type strain genome server.

The DDBJ/ENA/GenBank accession numbers for the 16S rRNA gene and genome sequences of strain PRKS01-65 are MK503548 and WYCT00000000, respectively. The version of whole genome sequence described in this paper is version WYCT01000000. The five housekeeping genes of strain PRKS01-65 used in the MLSA study were deposited in GenBank under the following accession numbers: atpD (MN938914), gyrB (MN938915), recA (MN938916), rpoB (MN938917) and trpB (MN938918).

Six supplementary figures and three supplementary tables are available with the online version of this article. $004346 \odot 2020$ The Authors 
Table 1. Cellular fatty acids (\%) of isolate PRKS01-65 and Streptomyces leeuwenhoekii $\mathrm{C} 34^{\mathrm{T}}$

-, Not detected; Data for the S. leeuwenhoekii strain was taken from Busarakam et al. [14].

\begin{tabular}{|c|c|c|}
\hline Fatty acid & $\begin{array}{c}\text { Isolate } \\
\text { PRKS01-65 }^{\mathrm{T}}\end{array}$ & $\begin{array}{c}\text { Streptomyces } \\
\text { leeuwenhoekii } \mathrm{C} 34^{\mathrm{T}}\end{array}$ \\
\hline \multicolumn{3}{|l|}{ Branched } \\
\hline antesio- $\mathrm{C}_{15: 0}$ & 22.9 & 29.2 \\
\hline antesio- $\mathrm{C}_{17: 0}$ & 10.2 & 13.8 \\
\hline antesio- $C_{17: 1} \omega 9 c$ & 5.1 & 1.1 \\
\hline iso- $\mathrm{C}_{10: 0}$ & 1.1 & - \\
\hline iso- $\mathrm{C}_{11: 0}$ & 2.0 & - \\
\hline iso- $\mathrm{C}_{14: 0}$ & 7.4 & 4.3 \\
\hline iso- $\mathrm{C}_{15: 0}$ & 4.6 & 5.5 \\
\hline iso- $\mathrm{C}_{16: 0}$ & 36.6 & 12.5 \\
\hline iso- $\mathrm{C}_{17: 0}$ & 2.8 & 4.4 \\
\hline iso- $\mathrm{C}_{18: 0}$ & - & 0.9 \\
\hline iso- $\mathrm{C}_{16: 1}-\mathrm{H}$ & 2.8 & - \\
\hline \multicolumn{3}{|l|}{ Saturated } \\
\hline $\mathrm{C}_{14: 0}$ & - & 1.1 \\
\hline $\mathrm{C}_{16: 0}$ & 2.9 & 19.1 \\
\hline $\mathrm{C}_{17: 0}$ & - & 3.5 \\
\hline $\mathrm{C}_{17: 0}$ cyclo & 1.7 & - \\
\hline
\end{tabular}

rich source of new bioactive compounds [11, 12]. A case in point was the discovery of new natural products with anticancer and anti-microbial properties from Streptomyces leeuwenhoekii strains isolated from hyper-arid Atacama Desert soil $[10,13-16]$. The type strain of this species, isolate C $34^{\mathrm{T}}$, is especially gifted sensu Baltz [17] as it has a large genome $(7.8 \mathrm{Mb})$ that includes 31 biosynthetic gene clusters (BGCs) that mainly encode for unknown specialized metabolites [18]. Such gifted Streptomyces strains are pivotal in the search for new drug-leads using state-of-the-art technologies such as genome mining and metabolic engineering $[19,20]$.

The aim of the present study was to establish the taxonomic status of a Streptomyces strain, isolate PRKS01-65 ${ }^{\mathrm{T}}$, recovered from an arid Indonesian sandy soil sample and found to be closely related to the type strain of S. leeuwenhoekii. These strains were the subject of a polyphasic study underpinned by genomic data derived from whole genome sequences. The resultant datasets confirmed that the isolate and the S. leeuwenhoekii strain are closely related but belong to different species. Consequently, the isolate is considered to represent a new species of Streptomyces for which the name Streptomyces harenosi sp. nov. is proposed. The type strain,
PRKS01-65 ${ }^{\mathrm{T}}\left(=\mathrm{NCIMB} 15211^{\mathrm{T}}=\mathrm{CCMM} B 1302^{\mathrm{T}}=\right.$ ICEBB $\left.03^{\mathrm{T}}\right)$, is a potential source of new natural products.

\section{ISOLATION, MAINTENANCE AND CULTIVATION}

Isolate PRKS01-65 $5^{\mathrm{T}}$ was recovered from an arid, non-saline soil sample ( $\mathrm{pH} 5.8$, organic matter content $0.06 \%$ ) collected just below the surface of a sand dune in the Parangkusumo region ( $8^{\circ} 1^{\prime} 7.513^{\prime \prime}$ S, $110^{\circ} 19^{\prime} 11.04^{\prime \prime}$ E) of Yogyakarta Province, Java, Indonesia by Ali Budhi Kusuma and his students in January 2013 (Fig. S1, available in the online version of this article). One gram of the soil sample was heated at $120^{\circ} \mathrm{C}$ for $15 \mathrm{~min}$, sprinkled directly over plates of actinomycete isolation agar (HiMedia), $\mathrm{pH} 7.3$, that were incubated at $45^{\circ} \mathrm{C}$ for up to 14 days [21]. Spores taken from a colony of isolate PRKS01-65 ${ }^{\mathrm{T}}$ growing on one of the isolation plates were used to inoculate yeast extract-malt extract agar [International Streptomyces Project (ISP) medium 2 [22]] plates., which were incubated at $28^{\circ} \mathrm{C}$ for $7-14$ days. Working cultures of the isolate and S. leeuwenhoekii $\mathrm{C} 34^{\mathrm{T}}[14]$ were maintained on ISP2 agar plates for long-term preservation the strains were kept as mixtures of hyphal fragments and spores in $20 \%, \mathrm{v} / \mathrm{v}$ glycerol at $-20^{\circ} \mathrm{C}$ and $-80^{\circ} \mathrm{C}$. Biomass for the chemotaxonomic analyses conducted on the isolate was harvested from ISP 2 broth cultures which had been shaken at 180 r.p.m. in baffled flasks for 14 days at $28^{\circ} \mathrm{C}$ following inoculation with $25 \mathrm{ml}$ seed culture of the isolate prepared under the same conditions. The harvested biomass was washed twice in sterile distilled water and freeze-dried.

\section{CHEMOTAXONOMIC, CULTURAL AND MORPHOLOGICAL PROPERTIES}

Isolate PRKS01-65 $5^{\mathrm{T}}$ was examined for chemotaxonomic, cultural and morphological properties known to be of value in Streptomyces systematics [4, 23]. Isomers of diaminopimelic acid $\left(\mathrm{A}_{2} \mathrm{pm}\right)$ were sought as described by Staneck and Roberts [24], whole-organism sugars after Lechevalier and Lechevalier [25], and isoprenoid quinones and polar lipid profiles following the integrated procedure of Minnikin and his colleagues [26]. Fatty acids extracted from the isolate, cultivated under the same conditions as in an earlier study on S. leeuwenhoekii C34 ${ }^{\mathrm{T}}$ [14], were methylated and analysed using the Sherlock Microbial Identification (MIDI) system and the resultant peaks identified using the ACTINO6 database [27]. Gram-stain and micromorphological properties were recorded following growth on ISP2 agar for 7 days at $28^{\circ} \mathrm{C}$. Growth taken from the ISP2 agar plate was examined for spore-chain arrangement and spore- surface ornamentation using a scanning electron microscope (Tescan Vega 3 , LMU instrument) following the procedure described by O'Donnell et al. [28]. Cultural properties of the isolate were recorded from tryptone-yeast extract, yeast extract-malt extract, oatmeal, inorganic salts-starch, glycerol-asparagine, peptone-yeast extract-iron and tyrosine agar plates (ISP media 1-7) [22] after 21 days at $28^{\circ} \mathrm{C}$. Aerial and substrate 
mycelial colours and those of diffusible pigments were determined by comparisons against colour charts [29].

The chemotaxonomic, cultural and morphological properties of the isolate were consistent with its classification in the genus Streptomyces [30]. The organism was Gram-stain-positive, formed circular colonies bearing a grey aerial spore mass (Fig. S2a) and an extensively branched substrate mycelium carrying aerial hyphae that differentiated into spiral chains of smooth surfaced spores (Fig. S2b). Whole-organism hydrolysates of the strain were rich in LL- $\mathrm{A}_{2} \mathrm{pm}$ (Fig. S3), galactose and ribose with lesser proportions of glucose and mannose (Fig. S4). The major isoprenologs were hexa- and octa-hydrogenated menaquinones ( 34 and $66 \%$, respectively) and the polar lipids included diphosphatidylglycerol, phosphatidylethanolamine (diagnostic component), phosphatidylinositol and phosphatidylinositol mannosides (Fig. S5). The major fatty acids were in very good agreement with those found in S. leeuwenhoekii $\mathrm{C} 34^{\mathrm{T}}$ [14], apart from differences in minor components, as exemplified by the absence of iso- $\mathrm{C}_{10: 0}, \mathrm{C}_{14: 0}$ and $\mathrm{C}_{17: 0}$ in the profile of isolate PRKS01-65 ${ }^{\mathrm{T}}$ (Table 1).

Table S1 shows that the isolate and S. leeuwenhoekii $\mathrm{C} 34^{\mathrm{T}}$ grew well on all of the ISP media. In general, the isolate formed a white to greyish-white aerial spore mass and a brown substrate mycelium on these media and the S. leeuwenhoekii strain an olivaceous greenish-grey aerial spore mass and a yellowishwhite substrate mycelium. A brown diffusible pigment was produced by the isolate on ISP media 1, 2 and 4 whereas S. leeuwenhoekii $\mathrm{C} 34^{\mathrm{T}}$ exhibited yellowish to yellowish-grey diffusible pigments on all the ISP formulations, apart from ISP media 1 and 5.

\section{WHOLE GENOME SEQUENCING}

Genomic DNA was extracted from wet biomass of a single colony of the isolate grown on ISP2 agar for 7 days at $28^{\circ} \mathrm{C}$ following the protocol provided by MicrobesNG (Birmingham, UK; www.microbesng.uk) and sequenced using a Miseq instrument (Illumina). Genomic DNA libraries were prepared at MicrobesNG using a Nextera XT library preparation kit. The purity and concentration of the extracted genomic DNA was measured using the Microlab STAR liquid handling system (Hamilton) and libraries determined with the Kapa Biosystem library quantification kit designed for Illumina instruments on a LightCycler 96 real time PCR instrument (Roche). The libraries were sequenced following the $2 \times 250$ bp paired-end protocol (MicrobesNG). Reads were trimmed using Trimmomatic software version 0.38 [31] and their quality assessed with in-house scripts and SAMtools, BedTools and BWA-MEM software [32-34]. Reads under 200 bp were discarded and contigs assembled using SPAdes software version 3.1.1 [35]. The draft genome assembly was annotated using the RASTweb server $[36,37]$ with default options and is available from GenBank (accession number WYCT00000000). The isolate was found to have a draft genome size of $8.0 \mathrm{Mb}$ with $175 \times$ mean coverage, 1096 contigs and 66 tRNA genes, a single 16S rRNA gene, $723 \mathrm{~S}$ rRNA genes, 7137 coding sequences (CDS) and an in silico $\mathrm{G}+\mathrm{C}$ content of $73 \mathrm{~mol} \%$. Genomic features of the S. leeuwenhoekii have been reported [18].

\section{PHYLOGENY}

An almost full-length 16S rRNA gene sequence (1528 bp; GenBank accession number MK503548) was extracted directly from the draft genome of the isolate using the ContEst16S tool available from the EZBioCloud webserver (www.ezbiocloud. net/tools/contest16s) [38]. The resultant 16S rRNA gene sequence was compared with corresponding sequences of the type strains of the most closely related Streptomyces species retrieved from the EzBiocloud server [39] following multiple sequence alignment generated using MUSCLE software [40]. Pairwise sequence similarities were determined using the single-gene tree option in the Genome-to-Genome Distance Calculator (GGDC) web server [41, 42]. Phylogenetic trees were inferred using the maximum-likelihood [43], maximumparsimony [44] and neighbour-joining [45] algorithms. The resultant trees were evaluated using bootstrap analyses based on 1000 replicates [46] from the MEGA X software package [47] using the two-parameter model of Jukes and Cantor [48]. The trees were rooted using a $16 \mathrm{~S}$ rRNA gene sequence from Streptomyces albus subsp. albus NRRL B-1811 ${ }^{\mathrm{T}}$ (GenBank accession number JX486031.1), the type strain of the type species of the genus Streptomyces.

The phylogenetic trees (Fig. 1) show that the isolate forms a well-supported branch in the Streptomyces 16S rRNA gene tree together with S. leeuwenhoekii $\mathrm{C} 34^{\mathrm{T}}$; the type strain of Streptomyces glomeratus [49] joins this group, albeit with low bootstrap support. Streptomyces chiangmaiensis TA4. $1^{\mathrm{T}}$ [50] is associated with this taxon but without statistical support. The isolate shares $16 \mathrm{~S}$ rRNA gene sequence similarities with these strains of $99.9,98.8$ and $98.9 \%$, respectively. The last two values are well below the threshold recommended by MeierKolthoff et al. [42] for assigning closely related actinobacteria to the same species. The isolate shares sequence similarities of either 98.7 or $98.8 \%$ with the remaining Streptomyces type strains. In general, these results correspond to those reported by Busarakam et al. [14], who noted that relationships between S. leeuwenhoekii $\mathrm{C} 34^{\mathrm{T}}$ and closely related Streptomyces species were sensitive to the treeing algorithms used, indicating that this part of the Streptomyces 16S rRNA gene tree is unstable.

Multilocus sequence analyses (MLSA) were undertaken based on 2528 nucleotides of partial sequences of five concatenated housekeeping genes: atpD (encodes ATP synthase F1 B-subunit), gyrB (gyrase B subunit), $\operatorname{rec} A$ (recombinase protein A), rpoB (DNA-directed RNA polymerase $B$ subunit) and $\operatorname{trp} B$ (tryptophan synthase $B$ subunit). The resultant MLSA tree (Fig. 2) was based on information taken from the draft genome of the isolate and from available corresponding partial gene sequences of Streptomyces strains accessed from the NCBI GenBank database, the sequence data are presented in Table S2. Pairwise sequence similarities between the datasets were calculated using the GGDC web server [41] and phylogenetic analyses conducted with the GGDC webserver and 


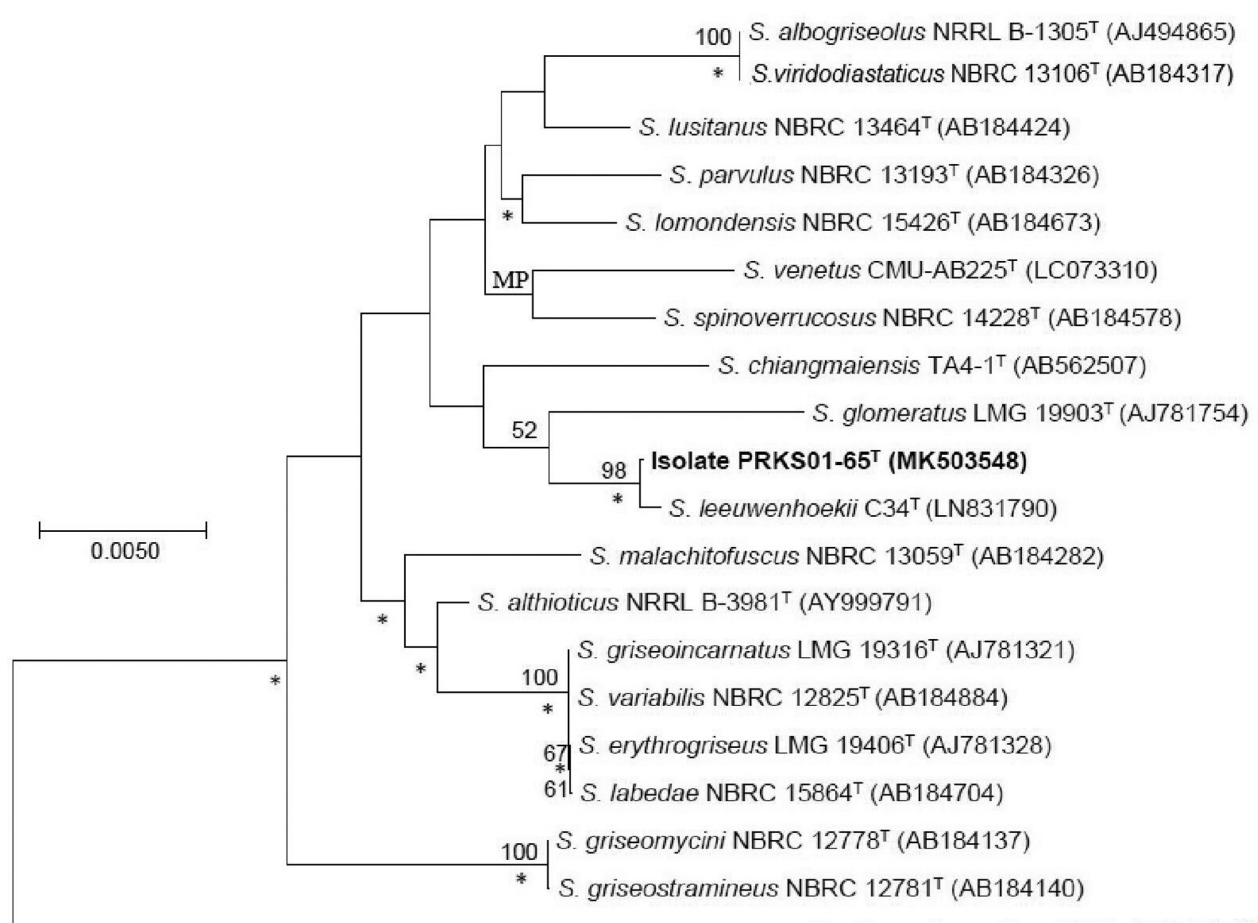

S. albus subsp. albus NRRL B-1811' (JX486031.1)

Fig. 1. Neighbour-joining tree based on $16 \mathrm{~S}$ rRNA gene sequences showing relationships between isolate PRKS01-65 ${ }^{\top}$ and the type strains of closely related Streptomyces species. Asterisks indicate branches of the tree that were found using the maximum-likelihood and maximum-parsimony algorithms. MP denote nodesrecovered using the maximum-parsimony tree-making algorithm. Numbers at the nodes indicate bootstrap values, only values above $50 \%$ are shown. The root of the tree was established using Streptomyces albus subsp. albus NRRL B-1811'. Bar, 0.005 substitutions per nucleotide position.

the DSMZ phylogenomic pipeline [51]. In turn, multiple sequence alignments were generated using MUSCLE software [40] and a maximum-likelihood tree inferred from alignment with RAxML [52] using rapid bootstrapping and the auto maximum-relative-error criterion [53]. In addition, a maximum-parsimony tree was inferred from the alignments with the Tree Analysis New Technology (TNT) program [54] using 1000 bootstraps with treebisection-and-reconnection branch swapping and 10 random sequence replicates. The sequences were checked

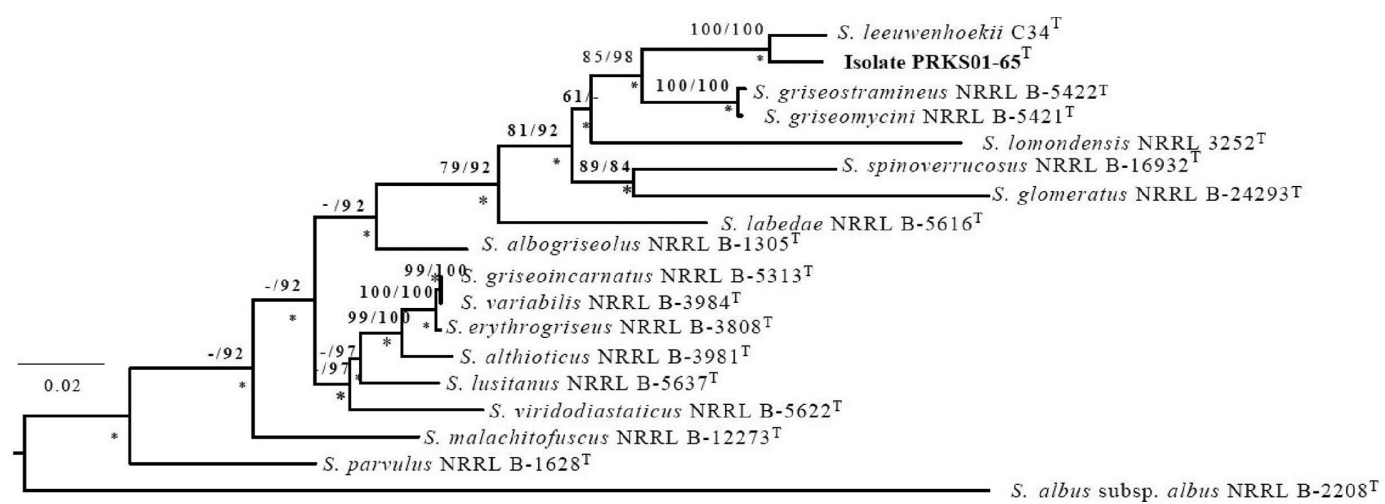

Fig. 2. Maximum-likelihood tree based on concatenated partial sequences of five housekeeping genes (2528 nucleotides) showing relationships between isolate PRKS01-65 and its closest phylogenetic neighbours. The tree was inferred using the GTR+GAMMA model. The branches are scaled in terms of the expected numbers of substitutions per site. The numbers above the branches are bootstrap support values of $60 \%$ or over from the maximum-likelihood (left) and maximum-parsimony (right) analyses. Asterisks indicate branches of the tree that were found using the neighbour-joining tree making-algorithm. Bar, 0.02 substitutions per nucleotide position. The root of the tree was established using Streptomyces albus subsp. albus NRRL B-2208'. 
for computational bias using the $\mathrm{X} 2$ test, as implemented in PAUP* (Phylogenetic Analysis Using Parsimony) [55].

It is apparent from the concatenated tree (Fig. 2) that most of the branches are supported by high bootstrap values providing further evidence that Streptomyces phylogenies based on MLSA gene sequences give better resolution than corresponding $16 \mathrm{~S}$ rRNA gene trees $[23,56]$. The MLSA tree underlines the close relationship between the isolate and S. leeuwenhoekii $\mathrm{C} 34^{\mathrm{T}}$ and shows that these strains are part to a well-defined clade that includes the type strains of Streptomyces griceomycini $[57,58]$, Streptomyces griseostramineus [57, 58] and Streptomyces lomondensis [59]., 60 the type strains of $S$. glomeratus [49], S. labedae [60] and Streptomyces spinoverrucosus [61] are located towards the periphery of this group. All the strains assigned to this clade produce spiral chains of spiny ornamental spores with the exception of isolate PRKS01-65 $5^{\mathrm{T}}$ and S. leeuwenhoekii $\mathrm{C} 34^{\mathrm{T}}$ [14], which form spiral chains of spores with smooth surfaces. The MLSA evolutionary distances between the isolates ranged from 0.016 to 0.141 (Table S3); that is, well above the species level threshold of $\leq 0.007$ used to distinguish between closely related species $[56,62]$. In the present analyses, the S. leeuwenhoekii strain was found to be related to a markedly different set of Streptomyces type strains when compared with corresponding MLSA data reported by Busarakam et al. [14] who found that relationships between the S. leeuwenhoekii and the type strains of closely related Streptomyces species varied depending on the treeing algorithm used. However, greater confidence can be placed in the results of the present analysis since most of the branches in the tree are supported by high bootstrap values.

A phylogenomic tree was generated based on whole-genome sequences of the isolate and its closest phylogenetic neighbors using the Type (Strain) Genome Server (TYGS) [63] available at http://tygs.dsmz.de. The minimum-evolutionary tree was inferred using FastME 2.1.6.1 software [64] based on the Genome BLAST Distance Phylogeny (GBDP). Distances were calculated from pairwise genome comparisons using formula d5 [41]. GBDP pseudo-bootstrap support values were calculated using 100 replicates and the tree rooted at the midpoint
[65]. The phylogenomic tree (Fig. 3) confirms the close relationship between the isolate and S. leeuwenhoekii $\mathrm{C} 34^{\mathrm{T}}$ as these strains form a well-supported branch in the phlogenomic tree that is sharply separated from corresponding branches composed of the type strains of the most closely related Streptomyces species.

\section{GENOMIC CHARACTERIZATION}

The genome sequence of isolate PRKS01-65 ${ }^{\mathrm{T}}$ was compared with that of S. leeuwenhoekii $\mathrm{C} 34^{\mathrm{T}}$ (GenBank accession number AZSD0000000). Orthologous average nucleotide identity (orthoANI) [66] and digital DNA-DNA hybridization $(\mathrm{dDDH})[42]$ values were calculated using the ANI calculator tool from the EZBioCloud (www.ezbiocloud.net/ tools/ani) and the GGDC webserver (http://ggdc.dsmz.de/ ggdc), respectively. The genome of the isolate was annotated and assigned to different functional classes using the RASTwebserver available at http://rast.nmpdr.org/

The $\mathrm{dDDH}$ similarities between the genome of the isolate and S. leeuwenhoekii $\mathrm{C} 34^{\mathrm{T}}$ was $56.0 \%$, a value well below the $70 \%$ threshold used to assign strains to the same genomic species [67]; the corresponding pairwise orthoANI value of $94.3 \%$ was just below the threshold used to distinguish between closely related prokaryotic species $[68,69]$. The relative distribution of the different functional gene classes in the genome of the isolate (Fig. S6) is similar to those found in the genomes of S. leeuwenhoekii C34, 'Streptomyces coelicolor' A3(2) [70] and 'Streptomyces lividans' 66 [71].

\section{PHENOTYPIC TRAITS}

Isolate PRKS01-65 $5^{\mathrm{T}}$ and $S$. leeuwenhoekii $\mathrm{C} 34^{\mathrm{T}}$ were examined for a broad range of phenotypic properties known to be value in distinguishing between Streptomyces species [8, 30, 72, 73]. The enzymatic profiles of the strains were determined using API-ZYM strips (bioMérieux) and biochemical, degradative and physiological properties using media and methods taken from Williams et al. [73]. The ability of the strains to grow under different temperature and $\mathrm{pH}$ regimes and in the

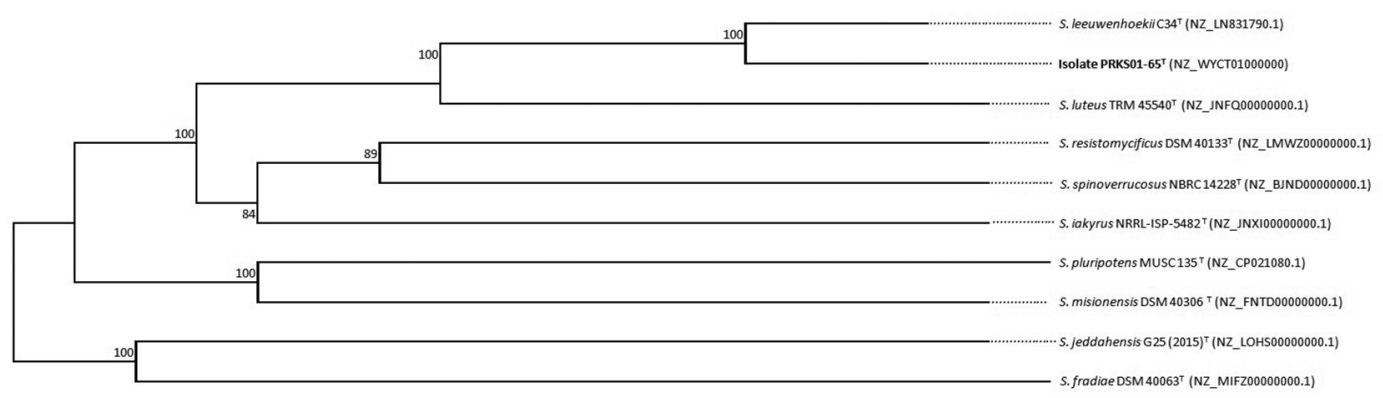

Fig. 3. Phylogenomic tree showing relationships between isolate PRKS01-65' and the most closely related Streptomyces type strains obtained using the TYGS platform. The number at the nodes are GBDP pseudo-bootstrap support values based on 100 replicates. The average branch support is $96.1 \%$. The tree is rooted at the midpoint. The accession numbers of genome sequences are given in parentheses. 
Table 2. Phenotypic properties that distinguish Streptomyces isolate PRKS01-65' from S. leeuwenhoekii $\mathrm{C} 34^{\top}$

+ , Positive result; -, negative result.

\begin{tabular}{|c|c|c|}
\hline \multirow[t]{2}{*}{ Characteristics } & Isolate & $\begin{array}{c}\text { Streptomyces } \\
\text { leeuwenhoekii }\end{array}$ \\
\hline & PRKS01-65 & $\mathrm{C} 34^{\mathrm{T}}$ \\
\hline \multicolumn{3}{|c|}{ Cultural characteristics on oatmeal agar } \\
\hline Aerial spore mass & Greyish-olive & Olivaceous grey-green \\
\hline Substrate mycelium & Greyish-olive & Yellowish-white \\
\hline Diffusible pigment & - & Yellowish \\
\hline \multicolumn{3}{|l|}{ API-ZYM tests } \\
\hline Alkaline phosphatase & - & + \\
\hline Esterase (C4) & - & + \\
\hline Lipase (C 14) & + & - \\
\hline a-Mannosidase & + & - \\
\hline \multicolumn{3}{|l|}{ Biochemical test } \\
\hline Urea hydrolysis & + & - \\
\hline \multicolumn{3}{|l|}{ Degradation tests } \\
\hline Adenine & - & + \\
\hline Chitin & + & - \\
\hline Elastin & - & + \\
\hline Guanine & - & + \\
\hline Tributyrin & - & + \\
\hline Tween 40 & - & + \\
\hline Xanthine & + & - \\
\hline \multicolumn{3}{|l|}{ Tolerance tests } \\
\hline $\mathrm{pH}$ range & $5.5-7.5$ & $6.0-11$ \\
\hline Temperature range $\left({ }^{\circ} \mathrm{C}\right)$ & $10-45$ & $4-50$ \\
\hline $\begin{array}{l}\text { Growth in presence of } \mathrm{NaCl} \\
(\%, w / v)\end{array}$ & $1-5$ & $1-10$ \\
\hline
\end{tabular}

presence of various sodium chloride concentrations were recorded using ISP2 agar as the basal medium. $\mathrm{pH}$ values were achieved using phosphate buffers. All of these tests were carried out in duplicate using a standard inoculum equivalent to 5.0 on the McFarland scale [74].

Identical results were obtained between the duplicated cultures for all of the phenotypic tests, several of which were weighted to distinguish between the isolate and S. leeuwenhoekii $\mathrm{C} 34^{\mathrm{T}}$ (Table 2). Thus, the isolate, unlike the $S$. leeuwenhoekii strain, produced $\beta$-glucosidase, lipase (C14) and $\alpha$-mannosidase, hydrolysed urea, and degraded chitin and xanthine. In contrast, only S. leeuwenhoekii $\mathrm{C} 34^{\mathrm{T}}$ formed alkaline phosphatase and esterase (C4), metabolized adenine, elastin, guanine, tributyrin and Tween 40 , and grew above $\mathrm{pH} 7.5$ and at 4 and $50^{\circ} \mathrm{C}$. Both strains produced acid phosphatase, $\alpha$-chymotrypsin, cystine arylamidase, esterase lipase, $\beta$-galactosidase, $\alpha$-acetyl- $\beta$-glucoaminidase, naphtholAS-BI-phosphohydrolase, trypsin and valine arylamidase, hydrolysed allantoin and arbutin, degraded hypoxanthine, starch, uric acid and Tween 80 , reduced nitrate, and were catalase-positive. Neither strain produced $a$-fucosidase, $\alpha$-glucosidase or $\beta$-glucoronidase, hydrolysed aesculin, or degraded casein, keratin, xylan or Tweens 20 and 60 .

The presence of putative biosynthetic gene clusters (BGs) encoding for natural products were sought in the genome of the isolate using the antiSMASH 5.0 platform [75] available at http://antismash.secondarymetabolites.org. AntiSMASH predicts BGCs and prospective products based on the percentage of genes from the closest known BGCs showing significant BLAST hits to the genome under consideration. In the present study, the genome of the isolate, unlike that of the S. leeuwenhoekii $\mathrm{C} 34^{\mathrm{T}}$, was associated with the production of several known antibiotics albeit with low gene similarities ( $<70 \%)$, as exemplified by actinoallolide A (30\%; [76]), enduracidin (6\%; [77]), rustimicin (6\%; [78]) and tautomycin $(27 \%$; [79]). In contrast, only the genome of S. leeuwenhoekii $\mathrm{C} 34^{\mathrm{T}}$ [18] contains the BGCs that express for a new family of ansamycin-like compounds, the chaxamycins, which show potent antibacterial and moderate anticancer activity [80].

It can be concluded from the present study that isolate PRKS01-65 $5^{\mathrm{T}}$ is a bone fide member of the genus Streptomyces. The isolate is closely related to S. leeuwenhoekii $\mathrm{C} 34^{\mathrm{T}}$, but not especially close to other Streptomyces species. The isolate and the $S$. leeuwenhoekii strain can be distinguished by a wealth of genotypic and phenotypic data, notably by a low $\mathrm{dDDH}$ value. It is proposed that isolate PRKS01-65 $\left(=\mathrm{NCIMB} 15211^{\mathrm{T}}=\mathrm{CCMM} \mathrm{B} 1302^{\mathrm{T}}=\mathrm{ICEBB}-03^{\mathrm{T}}\right)$ be recognized as the type and only strain belonging to Streptomyces harenosi sp. nov. The isolate has a large genome (8 Mb) which contains putatively novel BGCs and hence can be considered gifted in the sense of Baltz [17].

\section{DESCRIPTION OF STREPTOMYCES HARENOSI SP. NOV.}

Streptomyces harenosi (ha.re.no'si. L. gen. n. harenosi, of a sandy place referring to the source of the organism).

Aerobic, Gram-stain positive, catalase-positive actinobacterium which forms an extensively branched substrate mycelium that bears aerial hyphae which differentiate into spiral chains of spores $(0.8 \times 1.0 \mu \mathrm{m})$ with smooth surfaces on yeast extract-malt extract agar. Brown diffusible pigments are formed on tryptone-yeast extract, yeast extract-malt extract and inorganic salts-starch agar. Grows from 10 to $45^{\circ} \mathrm{C}$, optimally at $28^{\circ} \mathrm{C}$, from $\mathrm{pH} 5.5$ to 7.5 , optimally at $\mathrm{pH} 7.0$ and in the presence up to $5 \% \mathrm{w} / \mathrm{v}$ $\mathrm{NaCl}$. Allantoin, arbutin and urea are hydrolysed but not aesculin. Nitrate is reduced. Degrades chitin, hypoxanthine, starch, Tween 80, L-tyrosine, uric acid and xanthine, but not adenine, casein, elastin, keratin, Tween 20, tributyrin 
or xylan. Positive for acid phosphatase, $\alpha$-chymotrypsin, cystine arylamidase, esterase lipase, $\beta$-galactosidase, $\alpha$-acetyl- $\beta$-glucoaminidase, $\beta$-glucosidase, $\alpha$-mannosidase, naphthol-AS-BI-phosphohydrolase, trypsin and valine arylamidase, but negative for $\alpha$-fucosidase, $\alpha$-glucosidase and $\beta$-glucoronidase. Whole-organism hydrolysates are rich in LL- $\mathrm{A}_{2} \mathrm{pm}$, galactose and ribose, the predominant fatty acids are antesio- $\mathrm{C}_{15: 0}$ and iso- $\mathrm{C}_{16: 0}$, the major menaquinone is MK-9 (H8), and phosphatidylethanolamine is the diagnostic phospholipid. The DNA G+C content of the type strain is $73.36 \mathrm{~mol} \%$ and the approximate genome size $8.0 \mathrm{Mb}$.

The type strain, PRKS01-65 ${ }^{\mathrm{T}}\left(=\mathrm{NCIMB} 15211^{\mathrm{T}}=\mathrm{CCMM}\right.$ $\mathrm{B} 1302^{\mathrm{T}}=\mathrm{ICEBB}-03^{\mathrm{T}}$ ), was isolated from a sandy soil sample collected from an arid sand dune at Parangkusumo, Yogyakarta Province, Java, Indonesia. The GenBank accession number of the assembled draft genome of strain PRKS01-65 is WYCT00000000.

\section{Funding information}

A.B.K. is grateful for financial support awarded through the PhD Scholarship Scheme of the Indonesian Endowment Fund for Education (LPDP), Ministry of Finance, Indonesia. I.N. is grateful to Newcastle University for providing a postdoctoral fellowship and M.G. for an Emeritus Fellowship from the Leverhulme Trust.

\section{Acknowledgements}

Thanks are due to Mr. Ja'far Abdurrahman and Mr. Faiz Muhammad for helping to collect the composite environmental sample. We are indebted to Dr. Arief Budi Wietarto and Mr. Izzul Islam for their encouragement and support, to Mr. Piotr Czerniejewski for his help in Linux scripting, and to Professor Aharon Oren (The Hebrew University of Jerusalem, Israel) for suggesting the species name of the organism.

\section{Author contributions}

M.G., H.-P.K. and A.B.K designed the study, M.G and A.B.K prepared the manuscript. A.B.K. helped to collect the soil sample, characterized the strain under the supervision of I.N and M.G and deposited it in the culture collections. A.B.K. and I.N. were responsible for genome sequencing, annotation and the genome analyses. All of the authors approved the final version of the manuscript.

Conflicts of interest

The authors declare that there are no conflicts of interest.

\section{References}

1. Waksman SA, Henrici AT. The nomenclature and classification of the actinomycetes. J Bacteriol 1943;46:337-341.

2. Nouioui I, Carro L, Garcia-Lopez M, Meier-Kolthoff JP, Woyke T et al. Genome-based-taxonomic classification of the phylum Actinobacteria. Front Microbiol 2007;2018:9.

3. Labeda DP, Goodfellow M, Brown R, Ward AC, Lanoot B et al. Phylogenetic study of the species within the family Streptomycetaceae. Antonie van Leeuwenhoek 2012:101:73-104.

4. Labeda DP, Dunlap CA, Rong X, Huang Y, Doroghazi JR et al. Phylogenetic relationships in the family Streptomycetaceae using multi-locus sequence analysis. Antonie van Leeuwenhoek 2017:110:563-583.

5. Barka EA, Vatsa P, Sanchez L, Gaveau-Vaillant N, Jacquard C et al. Taxonomy, physiology, and natural products of actinobacteria. Microbiol Mol Biol Rev 2016;80:1-43.

6. Katz L, Baltz RH. Natural product discovery: past, present, and future. J Ind Microbiol Biotechnol 2016;43:155-176.

7. Zhu H, Swierstra J, Wu C, Girard G, Choi YH et al. Eliciting antibiotics active against the ESKAPE pathogens in a collection of actinomycetes isolated from mountain soils. Microbiology 2014;160:1714-1725.

8. van der Aart LT, Nouioui I, Kloosterman A, Igual J-M, Willemse $\mathrm{J}$ et al. Polyphasic classification of the gifted natural product producer Streptomyces roseifaciens sp. nov. Int J Syst Evol Microbiol 2019;69:899-908.

9. Goodfellow M, Fiedler H-P. A guide to successful bioprospecting: informed by actinobacterial Systematics. Antonie van Leeuwenhoek 2010;98:119-142.

10. Goodfellow M, Nouioui I, Sanderson R, Xie F, Bull AT. Rare taxa and dark microbial matter: novel bioactive actinobacteria abound in Atacama desert soils. Antonie van Leeuwenhoek 2018;111:1315-1332.

11. Bull AT, Goodfellow M. Dark, rare and inspirational microbial matter in the extremobiosphere: $16000 \mathrm{M}$ of bioprospecting campaigns. Microbiology 2019;165:1252-1264.

12. Sayed AM, Hassan MHA, Alhadrami HA, Hassan HM, Goodfellow M et al. Extreme environments: microbiology leading to specialized metabolites. J Appl Microbiol 2020;128:630-657.

13. Okoro CK, Brown R, Jones AL, Andrews BA, Asenjo JA et al. Diversity of culturable actinomycetes in hyper-arid soils of the Atacama desert, Chile. Antonie van Leeuwenhoek 2009:95:121-133.

14. Busarakam K, Bull AT, Girard G, Labeda DP, van Wezel GP et al. Streptomyces leeuwenhoekii sp. nov., the producer of chaxalactins and chaxamycins, forms a distinct branch in Streptomyces gene trees. Antonie van Leeuwenhoek 2014;105:849-861.

15. Abdelkader MSA, Philippon T, Asenjo JA, Bull AT, Goodfellow M et al. Asenjonamides $\mathrm{A}-\mathrm{C}$, antibacterial metabolites isolated from Streptomyces asenjonii strain KNN 42.f from an extreme-hyper arid Atacama desert soil. J Antibiot 2018;71:425-431.

16. Rateb ME, Ebel R, Jaspars M. Natural product diversity of actinobacteria in the Atacama desert. Antonie van Leeuwenhoek 2018;111:1467-1477

17. Baltz RH. Gifted microbes for genome mining and natural product discovery. J Ind Microbiol Biotechnol 2017;44:573-588.

18. Gomez-Escribano JP, Castro JF, Razmilic V, Chandra G Andrews B et al. The Streptomyces leeuwenhoekii genome: de novo sequencing and assembly in single contigs of the chromosome, circular plasmid pSLE1 and linear plasmid pSLE2. BMC Genomics 2015:16:485.

19. Ziemert $\mathrm{N}$, Alanjary $\mathrm{M}$, Weber $\mathrm{T}$. The evolution of genome mining in microbes - a review. Nat Prod Rep 2016;33:988-1005.

20. Sekurova ON, Schneider O, Zotchev SB. Novel bioactive natural products from bacteria via bioprospecting, genome mining and metabolic engineering. Microb Biotechnol 2019;12:828-844.

21. Idris H. Actinobacterial Diversity in Atacama Desert Habitats as a Road Map to Biodiscovery. PhD Thesis. Newcastle Upon Tyne, UK: Newcastle University,; 2016.

22. Shirling EB, Gottlieb D. Methods for characterization of Streptomyces species. Int J Syst Bacteriol 1966;16:313-340.

23. Goodfellow M, Busarakam K, Idris $\mathrm{H}$, Labeda DP, Nouioui I et al. Streptomyces asenjonii sp. nov., isolated from hyper-arid Atacama desert soils and emended description of Streptomyces viridosporus Pridham etal. 1958. Antonie van Leeuwenhoek 2017;110:1133-1148.

24. Staneck JL, Roberts GD. Simplified approach to identification of aerobic actinomycetes by thin-layer chromatography. Appl Microbiol 1974:28:226-231.

25. Lechevalier MP, Lechevalier H. Chemical composition as a criterion in the classification of aerobic actinomycetes. Int J Syst Bacteriol 1970;20:435-443.

26. Minnikin DE, O'Donnell AG, Goodfellow M, Alderson G, Athalye M et al. An integrated procedure for the extraction of bacterial isoprenoid quinones and polar lipids. J Microbiol Methods 1984;2:233-241.

27. Sasser M. Identification of Bacteria by Gas Chromatography of Cellular Fatty Acids, vol. 101, MIDI Inc Technical Notes; 1990. p. 1.

28. O'Donnell AG, Falconer C, Goodfellow M, Ward AC, Williams E. Biosystematics and diversity amongst novel 
carboxydotrophic actinomycetes. Antonie van Leeuwenhoek 1993;64:325-340.

29. Kelly K. Color-Name Charts Illustrated with Centroid Colors. Chicago: Inter-Society Color Council-National Bureau of Standards; 1953.

30. Kämpfer Pet al. Genus 1. Streptomyces Waksman and Henrici 1943, 339 AL emend. Rainey, Ward-Rainey and Stackebrandt, 1997, 486, emend. Kim, Lonsdale, Seong and Goodfellow 2003b, 113, emend. Zhi, Li and Stackebrandt 2009, 600. In: Goodfellow M, Kämpfer P. Busse H-J, Trujillo ME, Suzuki K-I et al. (editors). The Actinobacteria Part B. Bergey's Manual of Systematic Bacteriology, volume 5, 2nd edn. New York: Springer; 2012. pp. 1455-1767.

31. Bolger AM, Lohse M, Usadel B. Trimmomatic: a flexible trimmer for Illumina sequence data. Bioinformatics 2014;30:2114-2120.

32. Li H, Handsaker B, Wysoker A, Fennell T, Ruan J et al. 1000 genome project data processing subgroup, the sequence Alignment/Map format and SAMtools. Bioinformatics 2009;25:2078-2079.

33. Quinlan AR, Hall IM. BEDTools: a flexible suite of utilities for comparing genomic features. Bioinformatics 2010;26:841-842.

34. Li H. Durbin R. Fast and accurate short read alignment with Burrows-Wheeler transform. Bioinformatics 2009;25:1754-1760.

35. Bankevich A, Nurk S, Antipov D, Gurevich AA, Dvorkin M et al. SPAdes: a new genome assembly algorithm and its applications to single-cell sequencing. J Compu Biol 2012;19:455-477.

36. Aziz RK, Bartels D, Best AA, DeJongh M, Disz T et al. The RAST server: rapid annotations using subsystems technology. BMC Genomics 2008;9:75

37. Overbeek R, Olson R, Pusch GD, Olsen GJ, Davis JJ et al. The SEED and the rapid annotation of microbial genomes using subsystems technology (RAST). Nucleic Acids Res 2014;42:D206-D214.

38. Lee I, Chalita M, Ha S-M, Na S-I, Yoon S-H et al. ContEst16S: an algorithm that identifies contaminated prokaryotic genomes using $16 \mathrm{~S}$ RNA gene sequences. Int J Syst Evol Microbiol 2017;67:2053-2057.

39. Yoon S-H, Ha S-M, Kwon S, Lim J, Kim Y et al. Introducing EzBioCloud: a taxonomically United database of $16 \mathrm{~S}$ rRNA gene sequences and whole-genome assemblies. Int J Syst Evol Microbiol 2017;67:1613-1617

40. Edgar RC. Muscle: multiple sequence alignment with high accuracy and high throughput. Nucleic Acids Res 2004;32:1792-1797.

41. Meier-Kolthoff JP, Auch AF, Klenk H-P, Göker M. Genome sequence-based species delimitation with confidence intervals and improved distance functions. BMC Bioinformatics 2013;14:60.

42. Meier-Kolthoff JP, Göker M, Spröer C, Klenk H-P. When should a DDH experiment be mandatory in microbial taxonomy? Arch Microbiol 2013;195:413-418.

43. Felsenstein J. Evolutionary trees from DNA sequences: a maximum likelihood approach. J Mol Evol 1981;17:368-376.

44. Fitch WM. Toward defining the course of evolution: minimum change for a specific tree topology. Syst Zool 1971;20:406.

45. Saitou N, Nei M. The neighbor-joining method: a new method for reconstructing phylogenetic trees. Mol Biol Evol 1987;4:406-425.

46. Felsenstein J. Confidence limits on phylogenies: an approach using the bootstrap. Evolution 1985;39:783-791

47. Kumar S, Stecher G, Li M, Knyaz C, Tamura K. MEGA X: molecular evolutionary genetics analysis across computing platforms. Mol Biol Evol 2018;35:1547-1549.

48. Jukes TH, Cantor CR. Evolution of Protein Molecules, 3. London: Academic Press; 1969. pp. 21-132.

49. Gause GF, Preobrazhenskaya TP. Validation List no. 22. Int J Syst Bacteriol 1986;36:573-576.

50. Promnuan Y, Kudo T, Ohkuma M, Chantawannakul P. Streptomyces chiangmaiensis sp. nov. and Streptomyces lannensis sp. nov., isolated from the South-East Asian stingless bee (Tetragonilla collina). Int J Syst Evol Microbiol 2013;63:1896-1901.

51. Meier-Kolthoff JP, Hahnke RL, Petersen J, Scheuner C, Michael V et al. Complete genome sequence of DSM 30083(T), the type strain (U5/41(T)) of Escherichia coli, and a proposal for delineating subspecies in microbial taxonomy. Stand Genomic Sci2014;10:2.

52. Stamatakis A. RAXML version 8: a tool for phylogenetic analysis and post-analysis of large phylogenies. Bioinformatics 2014;30:1312-1313.

53. Pattengale ND, Alipour M, Bininda-Emonds ORP, Moret BME, Stamatakis A. How many bootstrap replicates are necessary? J Comput Biol 2010;17:337-354.

54. Goloboff PA, Farris JS, Nixon KC. Tnt, a free program for phylogenetic analysis. Cladistics 2008;24:774-786.

55. Swofford D. PAUP*: Phylogenetic Analysis Using Parsimony ( ${ }^{*}$ and other methods). Version 4.0 B10. Sunderland: Sinauer Associates; 2002.

56. Rong $X$, Huang Y. Multi-Locus sequence analysis: taking prokaryotic systematics to the next level. Meth Microbiol 2014;41:221-251.

57. Preobrazhenskaya TP, Blinov NO, Ryabova IDGauze GF, Preobrazhenskaya TP, Kudirna ES, Blinov NO, Ryabova ID et al. (editors). Problems of Classification of Actinomycetes-Antagonists. Medgiz, Moscow: USSR: Government Publishing House of Medical Literature; 1957. pp. 1-398.

58. Pridham TG, Hesseltine CW, Benedict RG. A guide for the classification of streptomycetes according to selected groups; placement of strains in morphological sections. Appl Microbiol 1958;6:52-79

59. Johnson LE, Dietz A. Lomofungin, a new antibiotic produced by Streptomyces lomondensis sp. N. Appl Microbiol 1969;17:755-759.

60. Lacey J. Nomenclature of Saccharopolyspora erythraea Labeda 1987 and Streptomyces erythraeus (Waksman 1923) Waksman and Henrici 1948, and proposals for the alternative epithet Streptomyces labedae sp. nov. Int J Syst Bacteriol 1987;37:458.

61. Diab A, Al-Gounaim MY. Streptomyces spinoverrucosus, a new species from the air of Kuwait. Int J Syst Bacteriol 1982;32:327-331.

62. Rong X, Huang Y. Taxonomic evaluation of the Streptomyces hygroscopicus clade using multilocus sequence analysis and DNA-DNA hybridization, validating the MLSA scheme for Systematics of the whole genus. Syst Appl Microbiol 2012;35:7-18.

63. Meier-Kolthoff JP, Göker M. TYGS is an automated highthroughput platform for state-of-the-art genome-based taxonomy. Nat Commun 2019;10:2182.

64. Lefort V, Desper R, Gascuel O. FastME 2.0: a comprehensive, accurate, and fast distance-based phylogeny inference program. Mol Biol Evol 2015;32:2798-2800.

65. Farris JS. Estimating phylogenetic trees from distance matrices. Am Nat 1972;106:645-668.

66. Lee I, Ouk Kim Y, Park S-C, Chun J. OrthoANI: an improved algorithm and software for calculating average nucleotide identity. Int J Syst Evol Microbiol 2016;66:1100-1103.

67. Wayne LG, Moore WEC, Stackebrandt E, Kandler O, Colwell RR et al. Report of the AD hoc Committee on reconciliation of approaches to bacterial Systematics. Int J Syst Evol Microbiol 1987;37:463-464.

68. Richter M, Rosselló-Móra R. Shifting the genomic gold standard for the prokaryotic species definition. Proc Natl Acad Sci USA 2009;106:19126-19131.

69. Chun J, Rainey FA. Integrating genomics into the taxonomy and systematics of the Bacteria and Archaea. Int J Syst Evol Microbiol 2014;64:316-324.

70. Bentley SD, Chater KF, Cerdeño-Tárraga A-M, Challis GL, Thomson NR et al. Complete genome sequence of the model actinomycete Streptomyces coelicolor A3(2). Nature 2002;417:141-147.

71. Cruz-Morales P, Vijgenboom E, Iruegas-Bocardo F, Girard G, Yáñez-Guerra LA et al. The genome sequence of Streptomyces lividans 66 reveals a novel tRNA-dependent peptide biosynthetic system within a metal-related genomic island. Genome Biol Evol 2013:5:1165-1175.

72. Williams ST, Goodfellow M, Alderson G, Wellington EM, Sneath PH et al. Numerical classification of Streptomyces and related genera. J Gen Microbiol 1983;129:1743-1813. 
73. Idris H, Labeda DP, Nouioui I, Castro JF, Del Carmen MonteroCalasanz M, Montero-Calasanz MDC et al. Streptomyces aridus sp. nov., isolated from a high altitude Atacama desert soil and emended description of Streptomyces noboritoensis Isono et al. 1957. Antonie van Leeuwenhoek 2017;110:705-717.

74. Murray P, Barron E, Phaller M, Ternover J, Yolkken R. Manual of clinical microbiology. Mycopathologia 1999;146:107-108.

75. Blin K, Shaw S, Steinke K, Villebro R, Ziemert N et al. antiSMASH 5.0: updates to the secondary metabolite genome mining pipeline. Nucleic Acids Res 2019;47:W81-W87.

76. Inahashi $Y$, Iwatsuki M, Ishiyama A, Matsumoto A, Hirose $T$ et al. Actinoallolides A-E, new anti-trypanosomal macrolides, produced by an endophytic actinomycete, Actinoallomurus fulvus MK10-036. Org Lett 2015;17:864-867.
77. Higashide E, Hatano K, Shibata M, Enduracidin NK. A new antibiotic I. Streptomyces fungicidicus No. B5477, an enduracidin producing organism. J Antibiot 1968;21:126-137.

78. Mandala SM, Thornton RA, Milligan J, Rosenbach M, GarciaCalvo $\mathrm{M}$ et al. Rustmicin, a potent antifungal agent, inhibits sphingolipid synthesis at inositol phosphoceramide synthase. J Biol Chem 1998;273:14942-14949.

79. MacKintosh C, Klumpp S. Tautomycin from the bacterium Streptomyces verticillatus. another potent and specific inhibitor of protein phosphatases 1 and 2A. FEBS Lett 1990;277:137-140.

80. Castro JF, Razmilic V, Gomez-Escribano JP, Andrews B, Asenjo J et al. The 'gifted' actinomycete Streptomyces leeuwenhoekii. Antonie van Leeuwenhoek 2018;111:1433-1448

Five reasons to publish your next article with a Microbiology Society journal

1. The Microbiology Society is a not-for-profit organization.

2. We offer fast and rigorous peer review - average time to first decision is 4-6 weeks.

3. Our journals have a global readership with subscriptions held in research institutions around the world.

4. $80 \%$ of our authors rate our submission process as 'excellent' or 'very good'.

5. Your article will be published on an interactive journal platform with advanced metrics.

Find out more and submit your article at microbiologyresearch.org. 